\title{
Activity of chlorpromazine on nfa1 and Mp2CL5 genes of Naegleria fowleri trophozoites
}

\author{
Supathra Tiewcharoen ${ }^{1}$, Jundee Rabablert ${ }^{2}$, Dusit Worawirunwong ${ }^{3}$, \\ Titima Pratumsrikajorn ${ }^{2}$, Saksun limsangurai $^{2}$, Virach Junnu ${ }^{1}$, \\ ${ }^{1}$ Department of Parasitology, Faculty of Medicine Siriraj Hospital, Mahidol University, Bangkok, Thailand; \\ ${ }^{2}$ Department of Biology, Faculty of Science, Silpakorn University, Nakorn Phathom, Thailand; \\ *Corresponding Author: jundee@su.ac.th \\ ${ }^{3}$ National Institute of Health, Department of Medical Sciences, Nonthaburi, Thailand.
}

Received 14 January 2011; received 15 February 2011; accepted 28 February 2011.

\begin{abstract}
Amoeba treatment of patients suffering from primary amoebic meningoencephalitis caused by Naegleria fowleri has not been successful. Damaged morphology and effect on genes of $N$. fowleri as the result of its initial interaction with drug may provide clue to the success of treatment. In this study, we investigated the activity of chlorpromazine compared with amphotericin $B$ and voriconazole against $N$.fowleri Khon Kaen strain using cell based assay and molecular techniques. Scanning electron and light micrograph showed the drug interaction of treated amoebae with $0.098 \mathrm{ug} / \mathrm{ml}$ chlorpromazine was faster than $0.002 \mathrm{ug} / \mathrm{ml}$ amphotericin $B$ and 12.5 $\mathrm{ug} / \mathrm{ml}$ of voriconazole. The morphological characteristics of treated amoebae with Gomori's trichrome stain correlated to the scanning electron microscope study. The effect of drugs to nfa1 and Mp2CL5 genes of treated amoebae found that at $120 \mathrm{~min}$ post exposure, chlorpromazine, voriconazole inhibited both genes except amphotericin B. Most of drug inhibited nfa1 except fluconazole. The results evaluated that chlorpromazine was higher potency and rapidly activity than amphotericin $B$ and voriconazole against $N$. fowleri trophozoites.
\end{abstract}

Keywords: Naegleria fowleri; Chlorpromazine; Scanning Micrograph; Gene Expression

\section{INTRODUCTION}

Naegleria fowleri, amoebo-flagellate free-living, is the causative agent of primary amebic meningoencephalitis (PAM) in humans, a fatal disease of central nervous system that affects mainly children and young adults [1].
The sensitivity of drugs against $N$. fowleri has been studied since 1982 [2]. To date, there is no available drug for PAM [3]. The activity of drugs to morphological characteristic of the Naegleria trophozoites provided an over view understanding of the potential activity. Stains have been used for classify microorganisms; acid- fast stain, fluorochrome stain, gram stain Gormori’s trichrome stain is rapid and cost-effective information for preliminary diagnosis of infectious diseases. This technique is simple procedure that produce well-stained smear of intestinal protozoa, human cell, yeast cells, and artifact material [4]. The descriptive study on surface membrane of the amoebae, scanning electron microscope (SEM) has been established on free living amoeba [5]. SEM study on chemotherapeutic agents against $N$. fowleri revealed the obvious cell destruction. Previous study showed the micrographs of $N$. fowleri HB-1 treated for different period of time with purify amoebacin A12-B. The results demonstrated the characteristic of cell damaged; abnormal pseudopodia and the cell rounded up at 10 min then the amoebae cell membrane ruptured with the release of cytoplasmic material after 30 min of inoculation [6]. Cytopathology of pathogenic and nonpathogenic Naegleria species; $N$. australiensis, $N$. fowleri, $N$. gruberi, and $N$. lovaniensis for cultured rat neuroblastoma cells B-103 and the pathogenicity for B-103 mice were reported. All four species of Naegleria exhibited surface extensions termed food cups by electron microscope including either $N$. australiensis or $N$. lovaniensis with B-103 cells established the cytopathology involved lysis of the B-103 target cells [7]. SEM has also been studied on cytopathogenesis of $N$. fowleri in humam neuroblastoma SK-N-MC cells [8]. The drug activity correlated between morphological characteristic and gene expression of trophic amoebae should be explained the detail of drug interaction.

It has been reported that $n f a 1$ and $M p 2 C L 5$ genes 
were use for distinguish pathogenic $N$. fowleri in water and soil samples $[9,10]$ which both of them; localized to the pseudopodia apparatus [11] and a unique membrane respectively $[12,13]$. In addition the function of $n f a l$ and Mp2CL5 genes were responsible for ingestion of cells during feeding and cells proliferation [14]. Many investigators reported the effect of drugs against $N$. fowleri using in vitro and in vivo $[15,16]$. However, no report has been studied the effect of drugs against morphology and virulent genes of $N$. fowleri. In present study, we focus on series morphological damage as well as effect of gene expression of treated amoebae. The purpose of this studies was to explore the effect of chlorpromazine and voriconazole which were compared with amphotericin B against $N$. fowleri in both morphogenesis and gene expression. This study should be either initially demonstrated on a series destruction of trophic amoebae or inhibited pathogenic gene regulation.

\section{METERIALS AND METHODS}

\subsection{Amoebae Cultivation}

Cultivation of free living $N$. fowleri (Khon-Kaen strain) trophozoites was cultured in $75-\mathrm{cm} 2$ tissue culture flask containing Nelson's medium supplemented with 5\% heat inactivated fetal calf serum (FCS) without antibiotics at $37^{\circ} \mathrm{C}$. The trophozoite pellet was harvested and kept at $4^{\circ} \mathrm{C}$ for $10 \mathrm{~min}$, then centrifuged at $5,000 \times \mathrm{g}$ for $10 \mathrm{~min}$. The pellets were resuspended in $5 \mathrm{ml}$ fresh culture medium and the number of viable cells in each preparation was determined by using $0.4 \%$ trypan blue exclusion method [17].

\subsection{Pharmacological Agents}

Powders of amphotericin B (Advanced Remedies Pvt, India), artesunate (Gulin Pharmaceutical, Guangxi, China), azithromycin (Ben Venue Laboratories, Ohio, USA), and voriconazole, (Vfend (R); Roerig/Pfizer Inc., NewYork, NY, USA) were dissolved in distilled water, as manufacture's instruction. Chlorpromazine (aqueous form, Government Pharmaceutical Organization, Bangkok, Thailand) and fluconazole (aqueous form Siam Bheasach, Bangkok, Thailand) were kept as manufacture's instruction.

\subsection{Effect of Single Drugs on N. Fowleri Growth}

The trophozoites $\left(5 \times 10^{6}\right.$ cells $\left./ \mathrm{ml}\right)$ were suspended in Nelson's medium in the presence or the absence of drugs at $10 \%$ of $\mathrm{IC}_{50}$, duplicates [13]. The samples were then incubated at $37^{\circ} \mathrm{C}$ for use in this experiment. Amphotericin B $0.002 \mathrm{ug} / \mathrm{ml}$, voriconazole $12.56 \mathrm{ug} / \mathrm{ml}$, flu- conazole $3.19 \mathrm{ug} / \mathrm{ml}$, azithromycin $0.67 \mathrm{ug} / \mathrm{ml}$, artesunate $0.39 \mathrm{ug} / \mathrm{ml}$, and chlorpromazine $0.098 \mathrm{ug} / \mathrm{ml}$ against amoebae trophozoites were performed in $1.5 \mathrm{ml}$ centrifuges tubes (Corning, USA) at $37^{\circ} \mathrm{C}$. All experiments were repeated three times. At the indicated times, trophozoites were harvested and centrifuged at $5000 \times g$ for $10 \mathrm{~min}$. The pellet was washed twice with $0.9 \%$ normal saline to stop reaction and centrifuged at $5000 \mathrm{~g}$ for $10 \mathrm{~min}$. The number of total cells was counted using Trypan blue exclusion method and observed under light microscope. The morphology changing of cells was stained with Gormori's trichrome and detected under light microscope as well as the ultrastructure surface membrane was investigated by scanning electron microscope. The remaining cell pellets were frozen at $-80^{\circ} \mathrm{C}$ for detection of gene expression. The effect of drugs in our experiment was derived from a previous report on these agents on $N$. fowleri.

\subsection{Morphological Study}

Untreated and treated trophozoites were smeared, fixed and stained with Gormori's trichrome staining, as previously described [18]. Morphological changes of the amoeba were observed under light microscope at 1, 3, 5 $\mathrm{h}$ after exposure. Untreated and treated trophozoites were also observed by scanning electron microscope. Briefly, the cells were pre-warmed $2.5 \%$ glutaraldehyde in $0.1 \mathrm{M}$ PBS, pH 7.3 at $4{ }^{\circ} \mathrm{C}$ for $3 \mathrm{~h}$ and post fixed with $1 \%$ osmium tetroxide (OsO4) in $0.1 \mathrm{M}$ PBS for $2 \mathrm{hr}$. After that, the specimens were dehydrated in a graded series of ethanol, critical point dried, mounted on to the metal stubs and coated with gold/palladium. Finally the specimens were examined and photographed under SEM (Hitachi S - 510) at an accelerating voltage of $25 \mathrm{kV}$. [8]

\subsection{Reverse Transcriptase Polymerase Chain Reaction (RT-PCR)}

Total RNA of the untreated and treated cell pellets at indicated times were extracted by Tri Reagent No TR 118 , as the manufacture described. Total RNA of the untreated and treated cell pellets were evaluated by RT-PCR using primers of $n f a 1$ [12] and Mp2CL5 [13] were followed as previously described. One $\mu \mathrm{g}$ of total RNA from each individual sample was used as a template to synthesize first strand cDNA using Maxime RT PreMix Kit (iNtRON Biotechnology, Korea), following manufacturer's instruction. Briefly, template RNA and RNase-free water were added into the MaximeRT PreMix tubes (Random primer) to a total volume of $20 \mu \mathrm{l}$, duplicate. The cDNA reaction was performed at $45^{\circ} \mathrm{C}$ for $60 \mathrm{~min}$ and at $95^{\circ} \mathrm{C}$ for $5 \mathrm{~min}$, respectively. The amplification was carried out in a $20 \mu \mathrm{l}$ total reaction volume by using 
Maxime PCR PreMix (iNtRON Biotechnology) with 50 pmol of forward and reverse primers and $20 \mu \mathrm{l}$ of template. The thermal profile of PCR was $94^{\circ} \mathrm{C}$ for $5 \mathrm{~min}$, followed by 35 cycles of $94^{\circ} \mathrm{C}$ for $30 \mathrm{~s}, 55^{\circ} \mathrm{C} 148$ for 30 $\mathrm{s}$, and $72^{\circ} \mathrm{C}$ for $45 \mathrm{~s}$ and a final extension cycle at $72^{\circ} \mathrm{C}$ for 5 min using Thermal cycler (DNA Engine® \& Peltier Thermal Cyclers, PCT-200, Bio-Rad, USA). Agarose gel electrophoresis PCR products were subjected to $1.5 \%$ agarose Trisborate-EDTA gel electrophoresis at $100 \mathrm{~V}$ for $30 \mathrm{~min}$. Gels were stained with ethidium bromide and visualization on a UV Tran illuminator at $260 \mathrm{~nm}$.

\subsection{Statistical Analysis}

Results were expressed as the mean \pm standard deviation error from three independent experiments carried out in triplicate. Student's $t$-test and the $P$-values of $<0.05$ was used to determine the level of significance.

\section{RESULTS}

\subsection{Light Microscopic Study}

Morphological changes of $N$. fowleri amoeba in the absence or presence of amphotericin B, voriconazole, fluconazole, artesunate, azithromicin, and chlorpromazine were observed. Untreated trophozoites was stained with Gomori's trichrome staining and observed under light microscope for $5 \mathrm{~h}$ post-treatment. The results revealed that viable trophozoites contained homogeneous green cytoplasm. Large nucleolus was located in central of nucleus and surrounded with completed nuclear membrane (Figure 1(a)). Most treated amoebae were clumped with course and fine hyperpigment granules. Trophozoites with $0.002 \mathrm{ug} / \mathrm{ml}$ amphotericin B showed cracking of surface membrane (Figure 1(b)) whereas trophozoites with $12.56 \mathrm{ug} / \mathrm{ml}$ voriconazole had numerous small dense granules inside cytoplasm but not observed nuclear membrane (Figure 1(c)). Amoebae trophozoites with 0.098 $\mathrm{ug} / \mathrm{ml}$ chlorpromazine showed increasing number of the vacuoles and developing sinus from fusion of food vacuole (Figure 1(d)). The other drugs showed slightly destructive morphology of trophozoites at indicated times.

\subsection{Scanning Electron Microscope Study}

SEM micrograph of trophozoites demonstrated wrinkle, sucker-like apparatus and binary fission (Figure 2(a)). Treated amoebae with amphotericin B showed subsequently decreasing of surface membrane, sucker-like apparatus, and binary fission during 1-3 h post treatment. Amphotericin B destroyed surface membrane and decreased a number of sucker apparatus, but increased wrinkle edema at $3 \mathrm{~h}$ post-treatment (Figures 2(b)-(d)). Chlorpromazine rapidly increased bleb formation at $1 \mathrm{~h}$ (Fig-

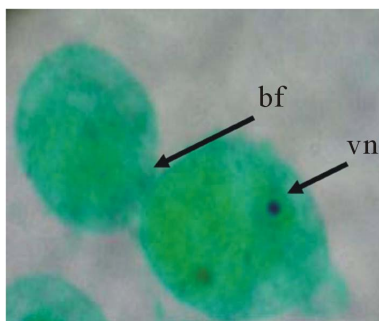

(a)

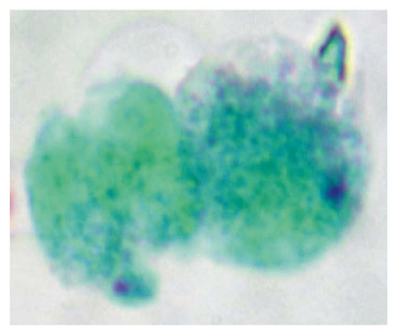

(c)

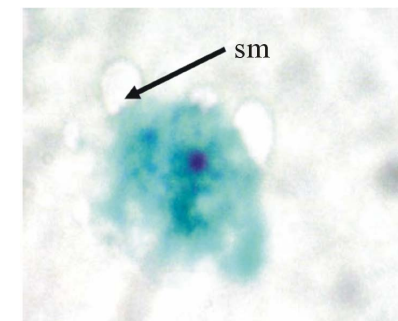

(b)

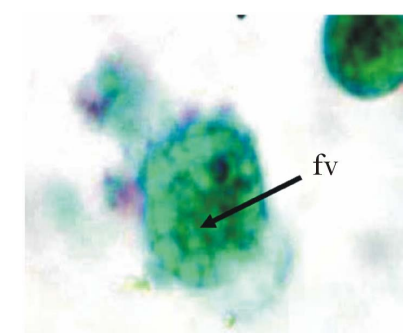

(d)
Figure 1. Light micrographs of $N$. fowleri Khon Kaen trophozoites with Gomori's trichrome stain in Nelson's media at $1 \mathrm{~h}$ showed binary fission and the cytoplasm was homogeneous green content. The vesicular nucleus showed normal characteristics (arrow) (a). The treated amoebae with $0.002 \mathrm{ug} / \mathrm{ml}$ amphotericin B showed cracking of surface membrane while vesicular nucleus was normal at $1 \mathrm{~h}$ (arrow) (b). At $3 \mathrm{~h}$, voriconazole (12.57 ug/ml)-treated amoeba, the cytoplasm was small dense granule inside and the nuclear membrane disappeared (c). At $0.098 \mathrm{ug} / \mathrm{ml}$ chlorpromazine, the small trophozoites was found with the appearance of dominant large size food vacuoles and the wall of a few food vacuoles were broken and merged into sinus (arrow) at $1 \mathrm{~h}$ (d). bf-binary fission, fvfood vacuole, sm-surface membrane, vn-vesicular nucleus $(\times$ 1,000).

ure 2(e)), and rough wrinkle (Figure 2(f)). However, chlorpromazine did not inhibit binary fission of the amoebae at $5 \mathrm{~h}$ post treatment. (Figure 2(g)). Slightly different morphology of $N$. fowleri trophozoites after treatment with fluconazole, voriconazole, artesunate and azithromicin compared with untreated trophozoites was observed at indicated times (data not shown). It was indicating that patterns of drug panel against $N$. fowleri were demonstrated by scanning electron microscope.

\subsection{Effects of Drugs on Naegleria Trophozoites at Gene Levels}

Generally, the expression of $n f a 1$ (360 bp) and Mp2CL5 genes (110 bp) of trophozoites were observed during proliferation growth. The activities of amphotericin B, voriconazole, fluconazole, artesunate, azithromycin, or chlorpromazine at 1/10 IC 50 against $n f a 1$ and Mp2CL5 genes of trophozoites were observed at 15, 30, 45, 60 and $120 \mathrm{~min}$, respectively, using RT-PCR . During 15-60 min, we found inhibition of $n f a l$ genes after treatment with amphotericin $\mathrm{B}$, voriconazole, fluconazole, azithro- 


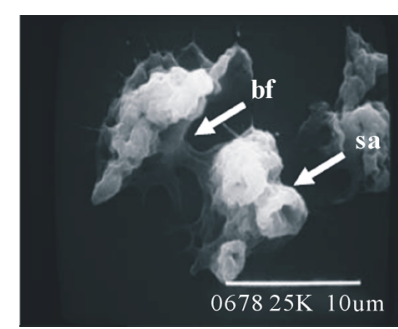

(a)

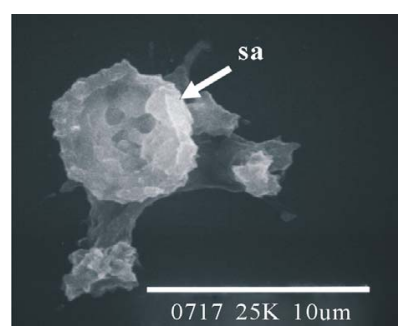

(b)

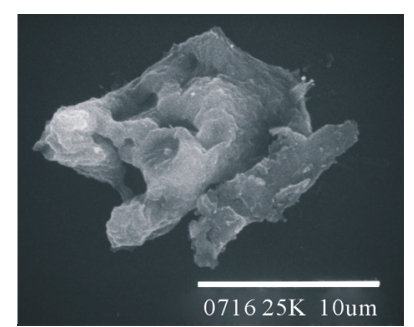

(c)

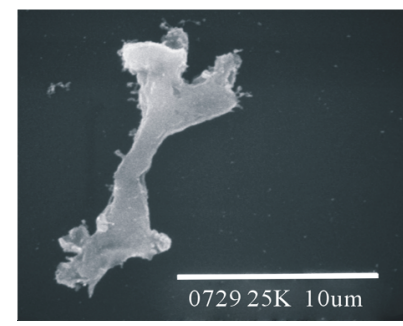

(d)

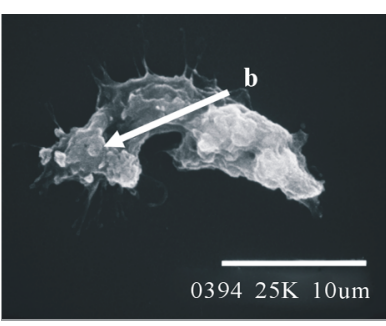

(e)

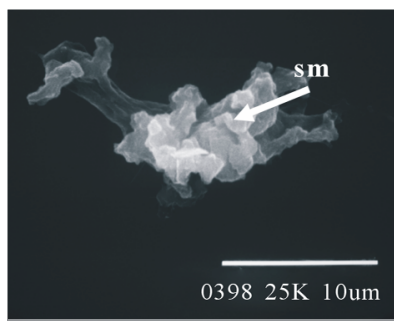

(f)

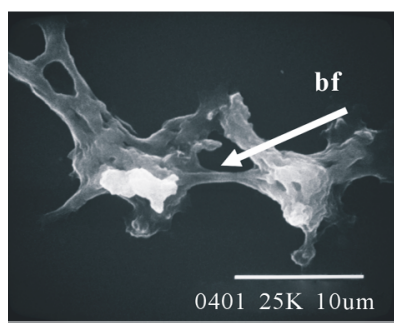

(g)

Figure 2. Scanning electron micrograph of $N$. fowleri trophozoite in the absence (a) or presence of amphotericin B (0.002 ug/ml) (b)-(d) or chlorpromazine at $0.098 \mathrm{ug} / \mathrm{ml}$ (e-g). Untreated trophozoites showed normal characteristics; equal binary fission and sucker apparatus (arrow) (a). Amphotericin B-treated amoebae showed sucker apparatus at $1 \mathrm{~h} \mathrm{(b).} \mathrm{Treated} \mathrm{amoebae} \mathrm{showed} \mathrm{the} \mathrm{destructive}$ surface membrane started at $3 \mathrm{~h}$; disappearance of sucker apparatus and edematous wrinkles surface (c). The series damage continued until wrinkles surface was not found (d). Chlorpromazine -treated amoebae showed the surface membrane was rapidly damage and the bleb formations were found (arrow) at $1 \mathrm{~h} \mathrm{(e).} \mathrm{The} \mathrm{destruction} \mathrm{of} \mathrm{amoebae} \mathrm{cell} \mathrm{was} \mathrm{continued;} \mathrm{sucker} \mathrm{and} \mathrm{pseudopodia} \mathrm{formation}$ were disappeared including the surface membrane showed edematous change at $3 \mathrm{~h}$ (arrow) (f). The unequal binary fission of the amoeba was seen at $5 \mathrm{~h}$ (arrow) (g). Bars represent $10 \mu \mathrm{m}$ : b-bleb formation, bf-binary fission, sa-sucker apparatus, sm-surface membrane, vn-vesicular nucleus.

mycin, artesunate, and chlorpromazine (data not shown). Furthermore, all drugs, except fluconazole inhibited $n f a 1$ genes at $120 \mathrm{~min}$ (Figure 3(a)). During 15-30 min, all drugs inhibited $m p 2 c 15$ gene expression of trophozoites (data not shown). From $30 \mathrm{~min}$ to $120 \mathrm{~min}$, voriconazole, fluconazole, and chlorpromazine also inhibited Mp2CL5 gene expression (Figure 3(b)), there by amphotericin B, azithromycin, and artesunate did not inhibit Mp2CL5 gene. It was indicating that the activities of drugs against $N$. fowleri trophozoites were time-dependent manner.

\section{DISCUSSIONS}

PAM is a severe infection of human that typically leads to death during 1 to 2 weeks from the onset of symptoms [19]. Amphotericin B is only drug of choice for PAM treatment [1]. However, it was a very toxic antibiotic and it caused renal toxicity, electrolyte disturbances, hematopoietic effects, and other organ damage, as well as chills, fever, nausea, vomiting, and headache [20]. In our study, we investigated the patterns of drugs against the trophozoites using light microscope and scanning electron microscope. Furthermore, we studied the activities of drugs against virulent $n f a 1$ and $M p 2 C L 5$ gene expression. This is the first report which demonstrated the characterization activity of drugs against amoeba trophozoites in both cellular and molecular levels. In the present study, light micrographs of $N$. fowleri
Khon Kaen trophozoites showed binary fission and showed normal characteristics of the vesicular nucleus. SEM micrograph of trophozoites demonstrated wrinkle, sucker-like apparatus and binary fission. Morphological characteristic of $N$. fowleri in this study was consistent with previous reports [8,21]. Morphology changing of trophozoites after drug treatment was revealed. Among these drugs, we found that the activity of chlorpromazine was higher potency than amphotericin B and voriconazole due to increasing number of the vacuoles, bleb formation and rough wrinkle at $1 \mathrm{~h}$. As previously described, our study correlated to the mechanism of chlorpromazine that acted on lipophilic action which effected on the plasma membrane and reflected sensitivity of amoeba calcium regulatory protein. Many researches revealed that $n f a 1$ and $M p 2 C L 5$ genes were found only pathogenic $N$. fowleri [10,11]. Nfa1 protein expressed from nfa1 11 gene was located in pseudopodia and around food vacuole [22]. Nfa1 protein was specifically localized food cups which are involved in phagocytic activity [11]. In this study, we found that most of drugs inhibited $n f a 1$ gene which represented that the amoebae should be loss of phagocytic activity Reveiller et al., demonstrated that the expression of native Mp2CL5 protein in $N$. flowleri appeared to be growth phase regulated [12]. The increased expression of the protein in the stationary phase of growth when the cells are experiencing nutrient 


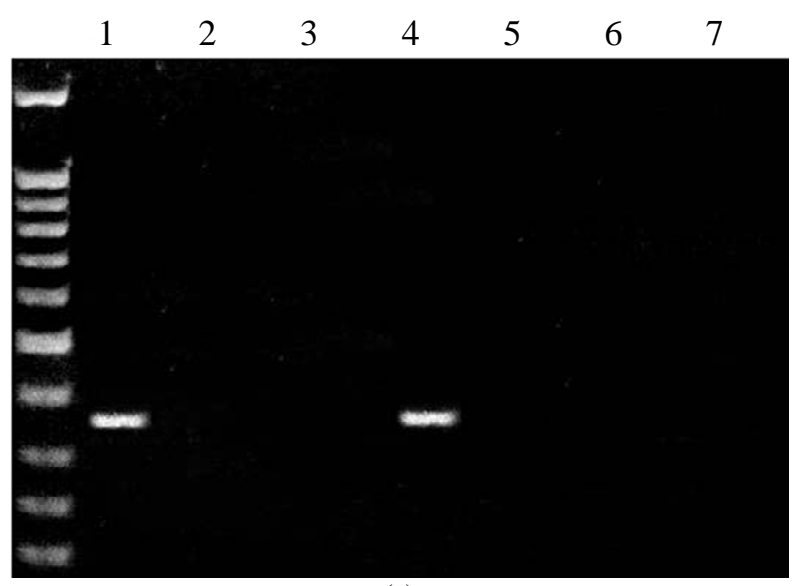

(a)

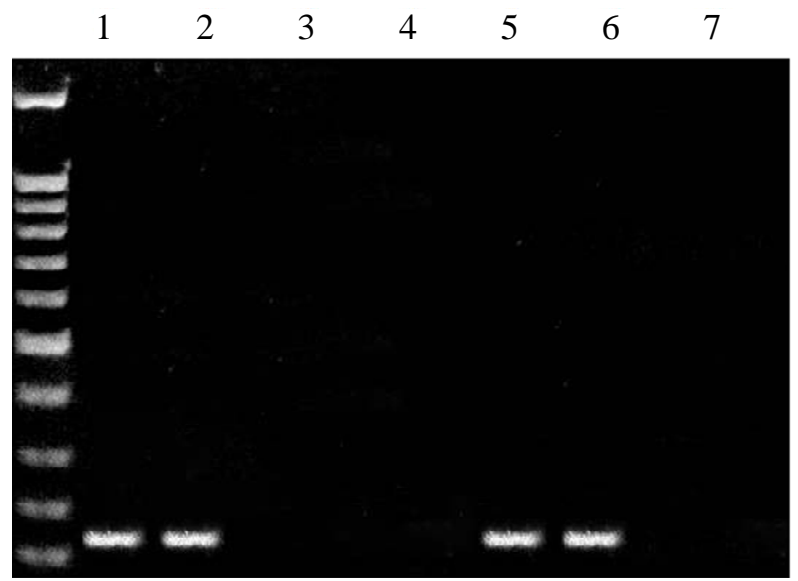

(b)

Figure 3. The $n f a 1$ (a) and Mp2CL5 (b) genes of $N$. fowleri trophozoites expressed in the absence or presence of drugs at 120 min post-treatment. Untreated $N$. fowleri (lane 1). $N$. fowleri treated with amphotericin B (lane 2), voriconazole (lane 3), fluconazole (lane 4), azithromycin (lane 5), artesunate (lane 6), and chlorpromazine (lane 7), respectively.

deprivation is consistent with our hypothesis that the protein may be involved in nutrient or environment sensing. In our study, we found that chlorpromazine and voriconazole inhibited Mp2CL5 gene, suggesting that activity of both drugs involved losing of cell recognition, sensing of environment and growth of amoeba. However, previously our report showed that at $\mathrm{MIC}_{100}$ amphotericin B and chlorpromazine inhibited trophic amoeba in 3 days but voriconazole did not [15]. Therefore, chlorpromazine should be proposed for treatment primary amoebic meningoencephalitis.

\section{CONCLUSIONS}

Our studies presented the correlation activity of drugs at ultrastructure and gene expressions of $N$. fowleri trophozoites. Chlorpromazine was rapidly damage surface membrane due to bleb formation occurred at 1 hour whereas the activity of amphotericin B was slow response at the same time in contrast it showed completely destruction during 5 hours. However both drugs could inhibit $n f a 1$ and Mp2CL5 gene of $N$. fowleri

\section{ACKNOWLEDGMENTS}

We are thank Associate Professor Dr. Darawan Wanachiwanawin, head of Department of Parasitology, Faculty of Medicine, Siriraj hospital, Mahidol University for providing the research facility. We also thank to Dr. Pathom awanpanyalert Director, National Institute of Health, Department of Medical Sciences. This work was supported by with Grant Number 017(II)50 from Faculty of Medicine, Siriraj Hospital, Mahidol University Foundation in 2007-2009 and partial fund from Grant Number RGP 2551/01 from Department of Biology, Faculty of Science, Silpakorn University at Sanamchan Palace, Nakhon Pathom, Thailand.

\section{REFERENCES}

[1] Schuster, F.L. and Visvesvara, G.S. (2004) Free-living amoebae as opportunistic and nonopportunistic pathogens of humans and animals. International Journal for Parasitology, 34, 1001-1027. doi:10.1016/j.ijpara.2004.06.004

[2] Ferrante, A. (1981) Comparative sensitivity of Naegleria fowleri to amphotericin B and amphotericin B methyl ester. Transactions of the Royal Society of Tropical Medicine and Hygiene, 76, 476-478. doi:10.1016/0035-9203(82)90141-9

[3] Soltow, S.M. and Brenner, G.M. (2007) Synergistic activities of azithromycin and amphotericin B against Naegleria fowleri in vitro and in a mouse model of primary amebic meningoencephalitis. Antimicrobial Agents and Chemotherapy, 51, 23-27. doi:10.1016/0035-9203(82)90141-9

[4] Madison, B.M. (2001) Application of stains in clinical microbiology. Biotechnic \& Histochemistry, 76, 119-125. doi:10.1016/j.exppara.2009.03.016

[5] González-Robles, A., Cristóbal-Ramos, A.R, GonzálezLázaro, M., Omaña-Molina, M. and Martínez-Palomo, A. (2009) Naegleria fowleri: Light and electron microscopy study. Experimental Parasitology, 122, 212-217.

[6] Gálvez, A., Valdivia, E., González-Segura, A., Lebbadi, M., Martínez-Bueno, M. and Maqueda, M. (1993) Purification, characterization, and lytic activity against Naegleria fowleri of two amoebicins produced by Bacillus licheniformis A12. Applied and Environmental Microbiology, 59, 1480-1486.

[7] Marciano-Cabral, F. and John, D. T. (1983) Cytopathogenicity of Naegleria fowleri for rat neuroblastoma cell cultures: scanning electron microscopy study. Infect Immunity, 40, 1214-1217.

[8] Tiewcharoen, S., Rabablert, J., Chetanachan, P., Junnu, V., Worawirounwong, D. and Malainual, N. (2008) Scanning electron microscope of Naegleria fowleri Thai strain during cytopathogenesis in human neuroblastoma cells. 
Parasitology Research, 103, 1119-1123. doi:10.1007/s00436-008-1103-4

[9] Maclean, R.C., Richardson, D.J., LePardo, R. and Marciano-Cabral, F. (2004) The identification of Naegleria fowleri from water and soil samples by nested PCR. Parasitology Research, 93, 211-217. doi:10.1007/s00436-004-1104-X

[10] Reveiller, F.L., Cabanes, P.A. and Marciano-Cabral, F. (2002) Development of a nested PCR assay to detect the pathogenic free-living amoeba Naegleria fowleri. Parasitology Research, 88, 443-450. doi:10.1007/s00436-002-0591-X

[11] Kang, S.Y., Song, K.J., Jeong, S.R., Kim, J.H., Park, S., Kim, K., Kwon, M.H. and Shin, H.J. (2005) Role of the Nfa1 protein in pathogenic Naegleria fowleri cocultured with CHO target cells. Clinical and Diagnostic Laboratory, 12, 873- 876.

[12] Reveiller, F. L., Suh, S., Sullivan, K., Cabanes, P.A and Marciano-Cabral, F. (2001) Isolation of a unique membrane protein from Naegleria fowleri. Journal of Eukaryotic Microbiology, 48, 676-682. doi:10.1111/j.1550-7408.2001.tb00208.x

[13] Madarova, L., Trnkova, K., Feiková, S., Klement, C. and Obernauerová, M. (2010) A real-time PCR diagnostic method for detection of Naegleria fowleri. Experimental Parasitology, 126, 37-41. doi:10.1016/j.exppara.2009.11.001

[14] Song, K.J., Jeong, S.R., Park, S., Kim, K., Kwon, M.H., Im, K., Pak, J. H. and Shin, H.J. (2006) Naegleria fowleri: Functional expression of the Nfa1 protein in transfected Naegleria gruberi by promoter modification. Experimental Parasitology, 112, 115-20. doi:10.1016/j.exppara.2005.10.004

[15] Tiewcharoen, S., Rabablert, J. and Junnu, V. (2009) In vitro susceptibility of Naegleria fowleri trophozoites to amphotericin B-combined chlorpromazine. Research Journal of Microbiology, 4, 320-333. doi:10.3923/jm.2009.320.333

[16] Kim, J.H., Jung, S.Y., Lee,Y.J., Song, K.J., Kwon, D., Park, S., Im, K.I. and Shin, H.J. (2008) Effect of therapeutic chemical agents in vitro and on experimental meningo-encephalitis due to Naegleria fowleri. Antimicrobial Agents Chemother, 52, 4010-4016. doi:10.1128/AAC.00197-08

[17] Tiewchreon, S., Malainual, N., Junnu, V., Chetanachan, P. and Rabablert, J. (2008) Cytopathogenesis of Naegleria fowleri Thai strains for cultured human neuroblastoma cells. Parasitology Research, 102, 997-1000. doi:10.1007/s00436-007-0866-3

[18] Gormori, G. (1950) A rapid one-step trichrome stain American Journal of Clinical Pathology, 20, 661-664.

[19] Shin, H. J. and Im, K. I. (2004) Pathogenic free-living amoebae in Korea. Korean Journal of Parasitology, 42, 93-119. doi:10.3347/kjp.2004.42.3.93

[20] Mandell, G. L. and Petri W. A. Jr (1996) Antimicrobial Agents. In: Hardman, J.G. and Limbird, L.E. eds., Goodman and Gilman's-The Pharmacological Basis of Therapeutics. McGraw-Hill, New York, US, 1165-1168.

[21] Antonios, S. N. (2010) Scanning electron microscopic study of trophozoite and cyst stages of Naegleria fowleri. Journal of the Egyptian Society of Parasitology, 40, 271-276.

[22] Shin, H.J., Cho, M.S., Jung, S.Y., Kim, H.I, Park, S. and Im, K.I. (2001) Molecular cloning and characterization of a gene encoding a $13 \cdot 1 \mathrm{kDa}$ antigenic protein of Naegleria fowleri. Journal of Eukaryotic Microbiology, 48, 713-717. doi:10.1111/j.1550-7408.2001.tb00211.x 